\title{
MR diffusion versus MR perfusion in patients with ovarian tumors; how far could we get?
}

Nadia F. El Ameen * (D), Mohamed G. Eissawy, Laila Adel M. S. Mohsen, Omnia Mokhtar Nada and Gerges M. Beshreda

\begin{abstract}
Background: Ovarian tumors are one of the prevalent gynecological tumors. They are the fifth commonest tumor in women. The therapeutic strategy depends on whether the tumor is benign, borderline or malignant. They always cause nonspecific symptoms at early stages and diagnosed at advanced stages. We tried to test and compare the sensitivity and accuracy of diffusion-weighted imaging (DWI) and MR perfusion in the diagnosis of ovarian tumors among fifty patients with ovarian masses who collected after meeting the inclusion criteria depending on grayscale sonographic findings after the approval of the ethical committee of our institution. The sensitivity and accuracy of the techniques were compared.

Results: The surgical and pathological reports of our patients proved a non-tumorous lesion in three patients. They also proved benign ovarian tumors in 18/47 (38.3\%) patients, borderline tumors in 4/47 (8.5\%) patients and malignant ovarian tumors in 25/47 (53.2\%) patients. According to conventional MRI, ovarian tumors could be diagnosed with sensitivity $92 \%$, specificity $61.11 \%$, PPV $76.7 \%$, NPV $84.6 \%$ and accuracy $79.1 \%$. DWI and MR perfusion showed the same results where they showed sensitivity $98 \%$, specificity $83 \%$, PPV 90\%, NPV 98\% and accuracy $93 \%$ with ADC cut-off value $1 \times 10-^{3} \mathrm{~mm}^{2} / \mathrm{s}$.
\end{abstract}

Conclusion: Diffusion-weighted imaging and MR perfusion are useful in the diagnosis of ovarian tumors with approximately the same sensitivity and positive impact on operative management.

Keywords: MRI ovarian cancer, MR diffusion, MR perfusion, Ovarian tumors characterization

\section{Background}

Ovarian tumors are the second prevalent gynecological tumors. They are the fifth commonest tumor in women and the leading indication for gynecologic surgery. The therapeutic strategy depends on whether the tumor is benign, borderline or malignant. Preoperative characterization is, therefore, crucial [1-3].

They always cause subtle and non-specific symptoms at early stages, and for this reason, ovarian cancer patients are usually diagnosed at advanced stages of the disease, which significantly impacts the treatment outcome and long-term survival. Thus, significant efforts are being directed at developing advanced diagnostic methods for a conclusive preoperative

\footnotetext{
* Correspondence: nadia.elameen@yahoo.com; nadia.elameen@hotmail.com Radiology Department, Faculty of Medicine, El Minia University, El Minia, Egypt
}

diagnosis of ovarian tumors to distinguish between benign and malignant phenotypes $[4,5]$.

MRI can be valuable as a problem-solving tool in surgical planning. Newly developed techniques of MRI such as diffusion-weighted imaging (DWI) and MRI perfusion (MRP) increased the technical capabilities for preoperative characterization and predicting the benign or malignant nature of ovarian masses $[6,7]$.

As a matter of fact, ovarian cancers are characterized by an anarchic neovascularization resulting in a wide number of immature microvessels. Angiogenic vessels have large gaps between the endothelial cells, the endothelium and the basement membranes as well as between the basement membrane and the pericytes making the vessels hyperpermeable to many macromolecules. These properties can be exploited by MRI perfusion. MR contrast agents that leak slowly through the normal vasculature are able to pass more quickly 
through tumor vessels to produce differential enhancement. This results in a fast "wash-in" of contrast coupled with the rapid "wash-out" and allows a functional analysis of the tumor microcirculation. These physio-pathogenic characteristics have been demonstrated to be in line with variations of MR perfusion parameters $[3,8]$.

In MRI perfusion, contrast agents are delivered intravenously via blood perfusion and leak into tumor interstitial space. The vascular volume and permeability is measured by monitoring the transport kinetics. Multi-parametric calculations of permeability and perfusion are used to characterize the tumor vasculature within a tumor "microenvironment". Thus, malignant lesions enhance in contrast quickly and have more intense signals, compared with benign lesions, due to the extensive vascular system associated with malignant tumors [9-11].

DWI is a non-invasive imaging modality depends on the Brownian motion of water molecules in tissues that is inversely correlated with cell density and cell membrane integrity which helps in discrimination between benign and malignant lesions. It increases the contrast between lesions and surrounding tissues and improves the detection and delineation of peritoneal implants at both initial staging and follow-up. Moreover, diffusionweighted imaging provides quantitative information about tissue cellularity that may be used to distinguish viable tumors from treatment-related changes [10].

\section{Aim of the work}

The aim of this study is to test and compare the sensitivity and accuracy of diffusion-weighted imaging (DWI) and MR perfusion (MRP) in women having ovarian tumors.

\section{Methods}

\section{Study design and population}

This study was a prospective study approved by the ethical committee of our institution. The study population comprised 50 consecutive women who were suspected to have ovarian neoplastic mass. Patients were collected between January 2018 and December 2018. Age ranged from 12 to 70 years with mean \pm SD of the age (39 \pm 15.9). They underwent MRI imaging after meeting the inclusion criteria based on the clinical history and grayscale transabdominal and or transvaginal sonography findings. All patients signed a written informed consent before MRI examination.

\section{Inclusion and exclusion criteria}

All patients included in this study had previous transabdominal or transvaginal real-time ultrasonography with diagnosis of complex ovarian lesions, cystic lesion with solid vegetation or thick septa or soft tissue component, solid ovarian lesions or suspicious adnexal lesions. Patients with simple cystic ovarian lesions, ovarian lesions with pure fatty component, impaired renal functions or general contraindications to MRI as the presence of any paramagnetic substances such as pacemakers, metallic clips or claustrophobic were excluded from the study (Fig. 1).

\section{Methods \\ Patient preparation}

Before MRI examination, all patients were routinely questioned about any conditions contraindicate MRI examination such as metallic implants, pacemakers, metallic clips or claustrophobics.

\section{MRI technique}

MR imaging was performed using 1.5 Tesla MR scanner (Ingenia, Philips Healthcare, Netherlands). All patients were imaged in the supine position using pelvic-phased array coil. The MRI examination was conducted on the female pelvis including conventional MRI sequences; axial, sagittal and coronal T2WIs using the following parameters (TR 5000/TE $110 \mathrm{ms,}$ echo train length 13-15, slice thickness 5-7 mm, gap $1-2 \mathrm{~mm}$, field of view $24-38 \mathrm{~cm}$, excitations (NSA) 3 and matrix 304.512). Followed by axial T1WI spinecho MR images (TR 162, TE 10-14 ms, slice thickness $5-7 \mathrm{~mm}$, gap $1-2 \mathrm{~mm}$, field of view $24-38 \mathrm{~cm}$, flip angle of $90^{\circ}$, excitations (NSA) $1-2$ and matrix 256 256).

Diffusion-weighted imaging was acquired in the axial plane prior to administration of contrast medium by using a single-shot echo-planar imaging sequence with $b$ values $(0,800,1000)$ (TR/TE $2871 / 78$, slice thickness $5 \mathrm{~mm}$, gap $1 \mathrm{~mm}$ ), FOV $375 \times 312 \times 161 \mathrm{~mm}$ and matrix $124 \times 105)$. All sequences were performed with saturation bands placed anteriorly to eliminate artifacts induced by the high signal from subcutaneous fat.

MRI perfusion was done for all patients using gadolinium chelate (dimeglumine gadopentetate) that was given at a dose of $0.2 \mathrm{ml}$ per kilogram of body weight by using a power injector (Medrad, spectris solaris $\mathrm{R}$ ) at a rate of $2 \mathrm{ml} / \mathrm{s}$, followed by $20 \mathrm{ml}$ of normal saline to flush the tubing. Images were obtained sequentially every $14 \mathrm{~s}$ beginning $14 \mathrm{~s}$ (first phase) before the bolus injection. A total of 40 sequential slices were taken with slice thickness of $2 \mathrm{~mm}$. Rapid acquisition (every 14 s) was performed for 30 consecutive phases with the whole time of the sequence $7 \mathrm{~min}$ to increase the accuracy of the early enhancement parameters and allows the derivation of accurate time-intensity curves. The images stack should include part of the soft 
a

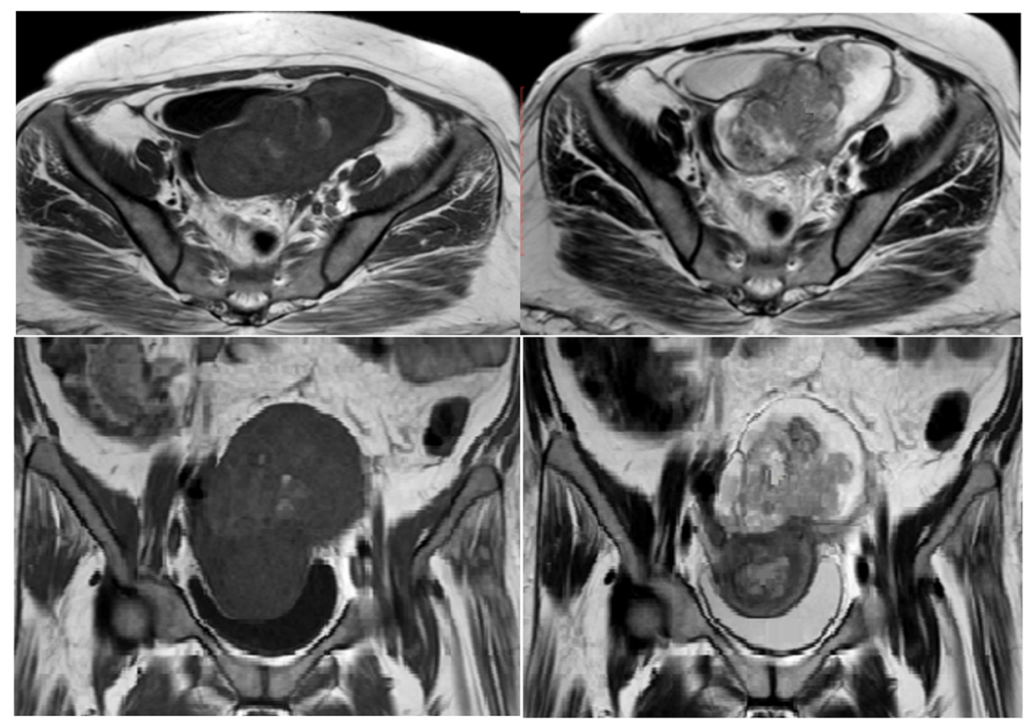

b

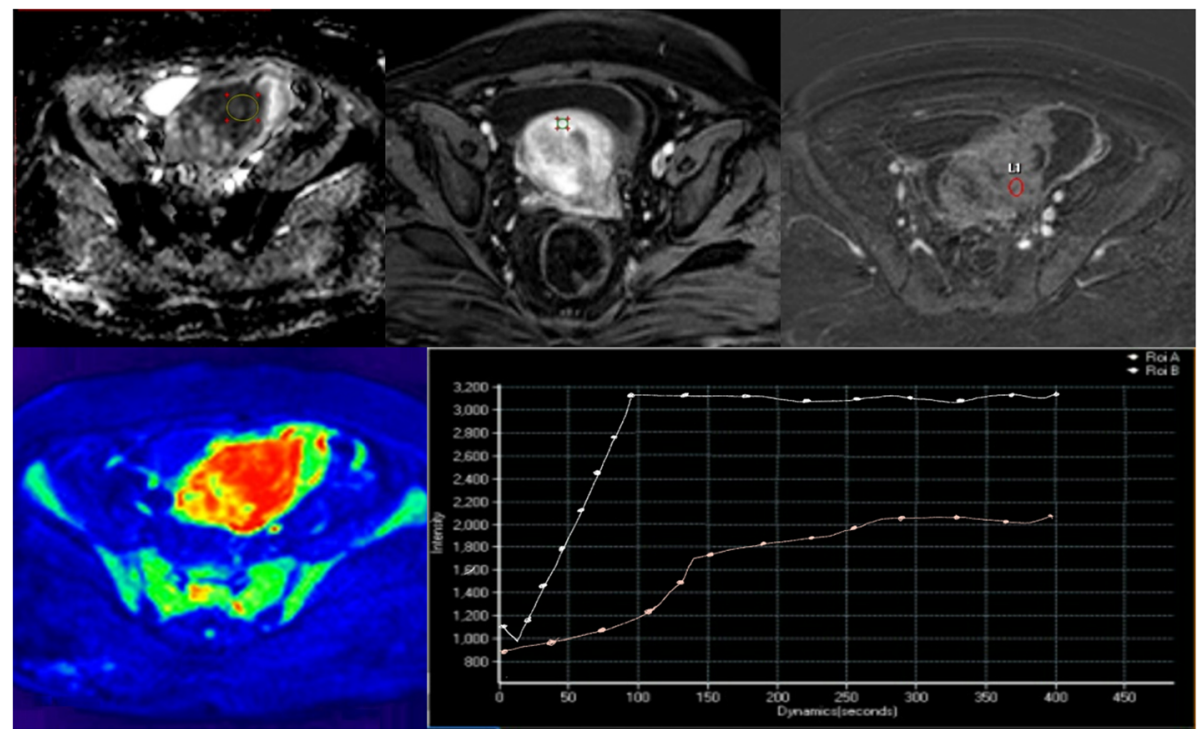

Fig. 1 A 52-year-old female complaining of pelvic pain and swelling. Ultrasonography revealed a large left cystic adnexal lesion with solid components. Axial and coronal T1 and T2 showed left adnexal cystic lesion with irregular soft tissue component. MR perfusion showed initial rapid enhancement followed by a plateau type 2 curve. Color map shows areas of maximum enhancement. Final histopathological diagnosis malignant mucinous cystadenocarcinoma

tissue inside the ovarian lesion (i.e., solid portion, papillary projections, or thickened irregular septa) and the adjacent external myometrium in the optimal plane. It is performed on the appropriate plane that shows the lesion and the myometrium on the same image, axial plane was used in most of the cases (Fig. 2).

\section{Data processing and image interpretation}

Images were transformed to Philips 881030 Intelli-Space IX/LX Workstation. Images were interpreted by three experienced radiologists NE, LA and MG. They have experienced of more than 10 years in MRI of female imaging. Final results were collected after the agreement of all three radiologists who already have an idea about the preliminary data of the previous ultrasonography of the patients. Each MR sequence findings were evaluated as following (Fig. 3):

(A) Analysis of conventional sequences

Conventional MRI sequences were evaluated for the morphologic features of the lesion including the lesion 


\section{a}

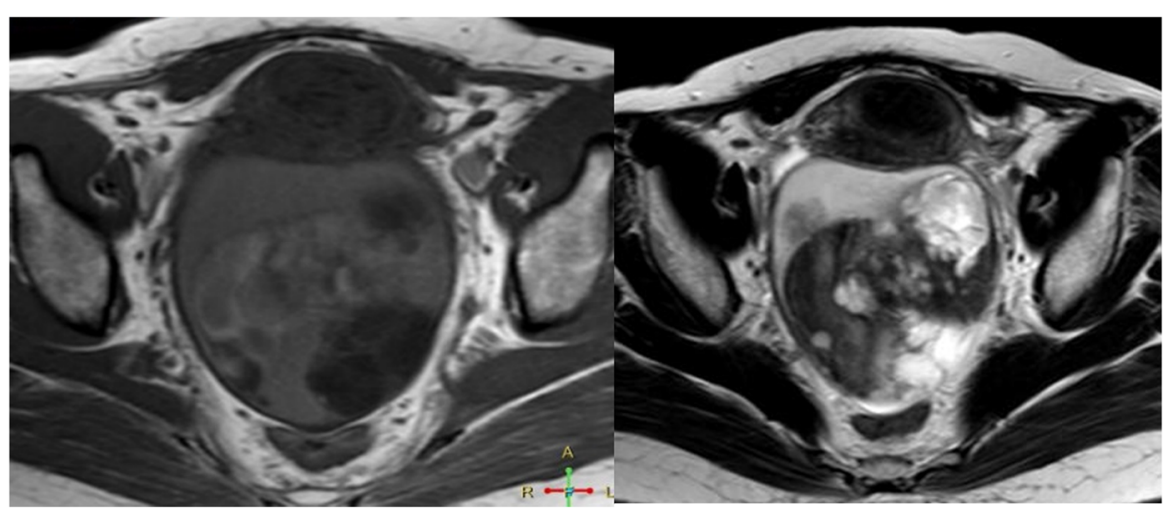

b

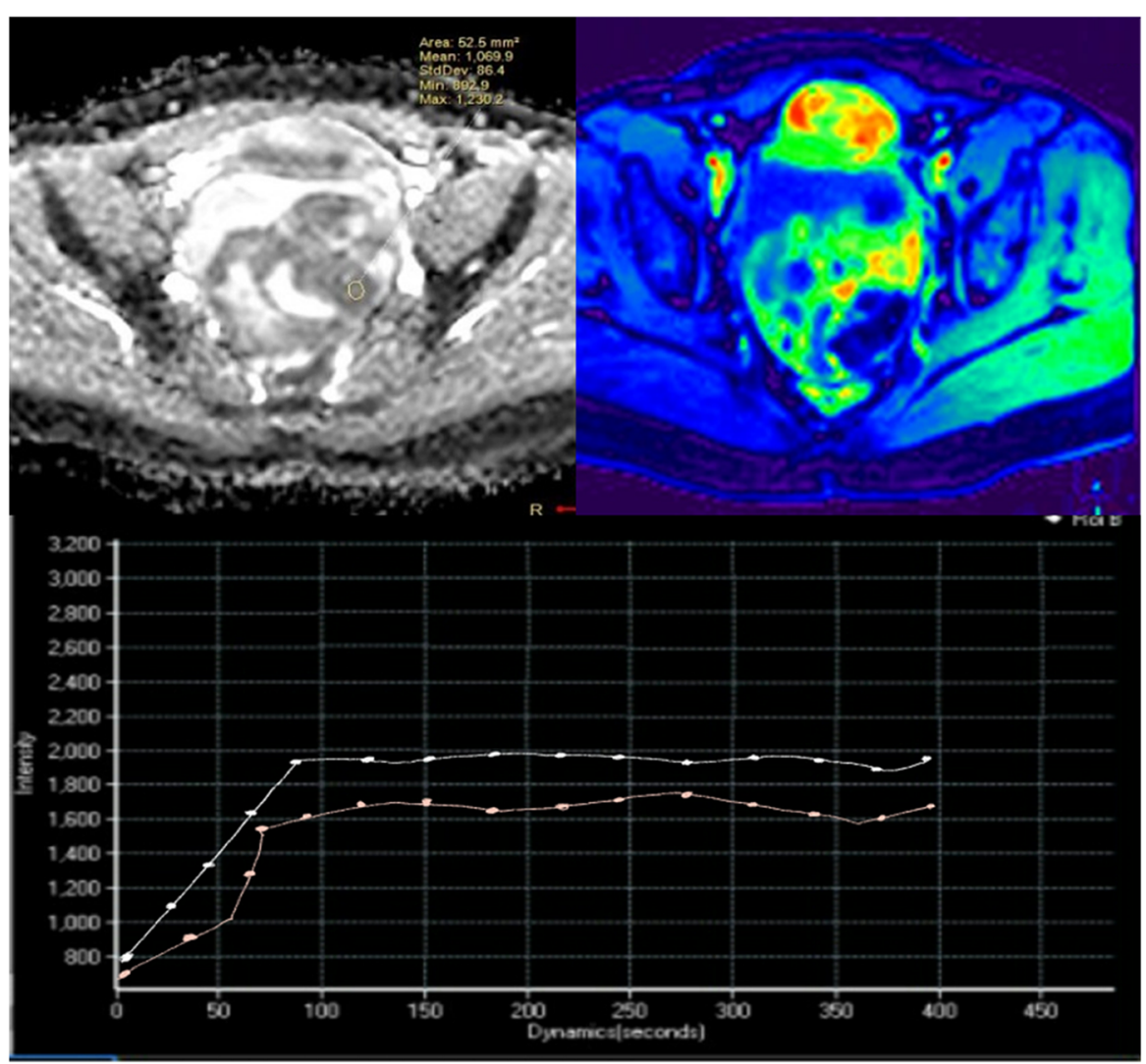

Fig. 2 A 28-year-old female presented with abdominal swelling. US revealed a large complex adnexal mass with thick septations and solid component. Axial T1 and T2 show a well-defined right adnexal lesion, dominantly cystic with thick septations and a small posterior solid component. MRI perfusion show slow rising enhancement type 1 curve. Color map shows the areas of maximum enhancement. Final histopathological diagnosis struma ovarii (benign ovarian cyst contain thyroid tissue)

laterality, size, shape, and complexity of the tumor, T2 signal intensity of the solid part inside the mass and the presence of ascites and peritoneal deposits. Sagittal T2WI was used for the best evaluation of endometrial thickness which may be associated with functioning ovarian tumors. Axial T2WI was used for anatomical localization of the lesion to depict the origin of the lesion (ovarian, uterine or broad ligament). Axial T1WI and fat saturation were evaluated for the presence of intralesional blood or fat.

(B) Analysis of diffusion-weighted images (DWI)

- Qualitative analysis 
a

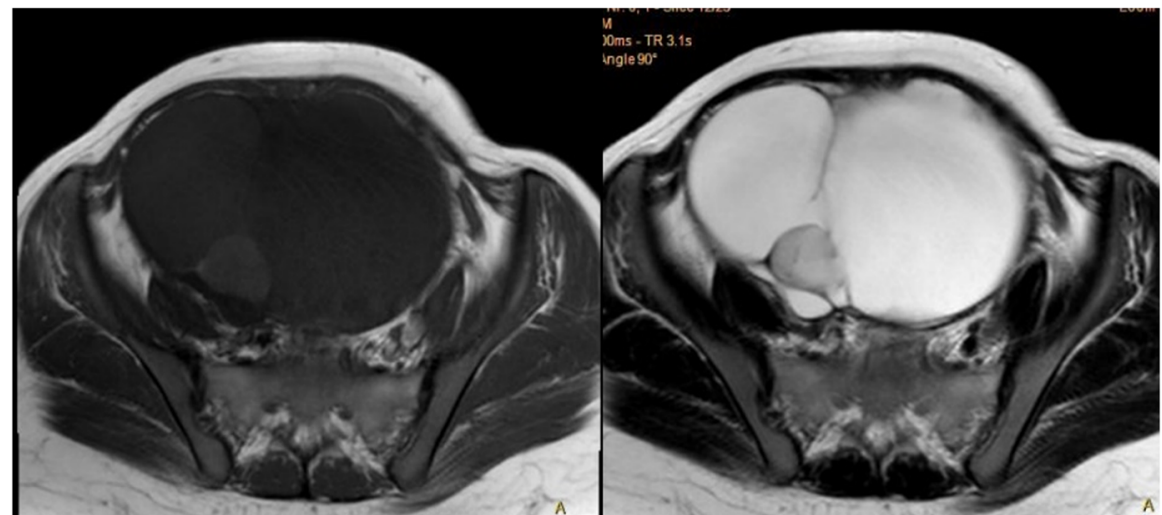

b

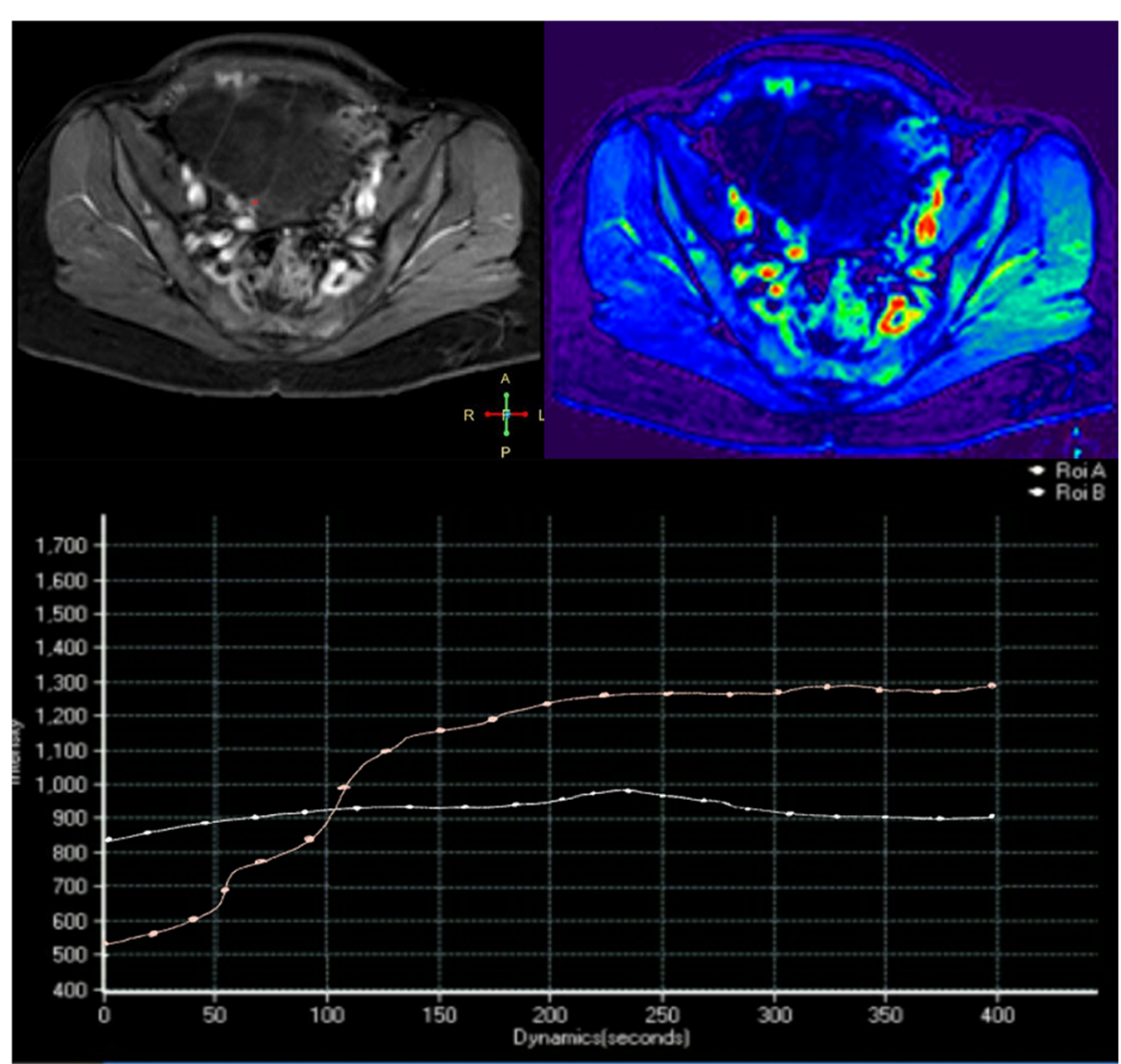

Fig. 3 A 45-year-old female presented with pelvic pain and pelvi-abdominal swelling. The patient underwent hysterectomy 5 years ago. US revealed a large complex right adnexal cystic mass with solid components. Axial T1 and T2 predominantly cystic right adnexal lesion with solid components within. Ascites and peritoneal deposits are seen. MR perfusion shows early intense increasing enhancement type 3 curve. Color map shows the areas of maximum enhancement. Final histopathological granulosa cell tumor

Regarding the signal intensity, lesions with low signal intensity on diffusion images and high signal in the corresponding ADC maps assigned for facilitated diffusion and diagnosed as benign masses. Lesions with high signal intensity on diffusion images with lowering of the signal in the corresponding ADC maps assigned for restricted diffusion and diagnosed as malignant masses.
- Quantitative analysis

Regarding the quantitative analysis of DWI, we generated the ADC map, then we selected the ROI manually on the solid and the cystic component of the tumors, which was then automatically calculated on the work station to get the ADC values. 
(C) Analysis of MRI perfusion

For ovarian tumor characterization, two regions of interest (ROI) were placed. One on the external myometrium and one on the most enhancing part of solid tissue of the ovarian mass. The most enhanced solid part was determined by the use of maximum enhancement colored generated map by the workstation. The enhancement of the solid tissue was classified by using a time-signal intensity curve classification:

1- A gradual increase in the signal intensity of the solid tissue, without a well-defined shoulder, was defined as type 1 curve (benign lesion curve).

2- A moderate initial increase in the signal intensity of the solid tissue relative to that of the myometrium followed by a plateau was defined as type 2 curve.

3- An initial increase in the signal intensity of the solid tissue that was steeper than that of the myometrium was defined as type 3 curve (malignant lesion curve).

Enhancement measures:

Maximum enhancement (SI max): Difference between peak intensity S1 and S0.

Maximum relative enhancement: (MRE) Maximum of all relative enhancements

$(\mathrm{SI}$ rel $)=(\mathrm{SI} \max -\mathrm{SI0}) / \mathrm{SI} 0 \times 100$.

T0-Time of arrival: Arrival of the contrast agent (begin of enhancement curve).

Time to peak: Time between $\mathrm{T} 0$ and the time of peak intensity.

Wash in rate (WIR): Maximum slope between T0 and the time of peak intensity T1 = SI0-SImax/time (s).

Wash-out rate (WOR): Maximum slope between the time of peak intensity T1 and the end of measurement.

Brevity of enhancement: Time between point of maximum wash in rate and maximum wash-out rate.

Area under the curve: Sum of all intensities under the curve.

From those quantitative data, only MRE, SI max, WIR and WOR were analyzed.

\section{Histopathological evaluation}

All of the patients underwent surgical management. Twenty-seven patients underwent radical hysterectomy with bilateral salpingo-oophorectomy, 5 cases underwent simple oophorectomy, 10 cases underwent ovarian cystectomy and 8 cases underwent simple oophorectomy. All results were correlated to final post-operative histopathological data which collected and correlated to the imaging findings of each sequence.

\section{Statistical analysis}

Statistical analysis was performed using the SPSS software for Windows v. 20 (SPSS Inc., Chicago, IL). Data were statistically described in terms of range, mean \pm $\mathrm{SD}$, frequencies (number of cases) and percentage when appropriate. For comparing quantitative data, KruskalWallis test and Mann-Whitney tests were performed. For comparing qualitative data, Fisher exact test was performed. The accuracy of the studied diagnostic test in predicting malignancy was represented using the terms sensitivity, specificity, overall accuracy, negative and positive predictive values. A probability value $(P=$ $0.05)$ was considered statistically significant. The receiver operating curve ROC was used to determine the cutoff values of the semi-quantitative parameters.

\section{Results}

Our study included 50 female patients, their ages ranged between 12 and 70 years with mean age $39 \pm 15.9$ year old. There was statistical significance between age and final pathological diagnosis $P$ values 0.001 for benign versus malignant lesions (Table 1 ).

Three patients were found to have non-ovarian tumors on histopathological evaluation, two of them were tuboovarian abscesses and one was cyst with hemorrhagic infarction. Those three cases were excluded from the study to avoid bias.

The epidemiological data showed that the main representing symptom was pelvic pain in 30/47 cases (63.8\%) (Table 2). There was a statistically significant difference between menopausal status and pathological diagnosis.

Table 1 Correlation between patient age and final pathological diagnosis of 47 patients with ovarian tumors

\begin{tabular}{|c|c|c|c|c|c|c|}
\hline & \multicolumn{3}{|c|}{ Pathological diagnosis } & \multirow[t]{3}{*}{$P$ value } & & \\
\hline & Benign (I) & Borderline (II) & Malignant (III) & & & \\
\hline & $N=18$ & $N=4$ & $N=25$ & & & \\
\hline \multicolumn{7}{|l|}{ Age } \\
\hline Range & $(16-57)$ & $(28-64)$ & $(12-70)$ & & & \\
\hline Mean \pm SD & $28.6 \pm 11.2$ & $47 \pm 14.8$ & $45.2 \pm 15.4$ & | vs || & | vs ||| & \| vs II \\
\hline Median & 27.5 & 48 & 50 & $0.045^{*}$ & $0.001^{*}$ & 0.825 \\
\hline
\end{tabular}

*Significant level at $P$ value $<0.05$ 
Table 2 Demographic data and clinical presentation of the patient's cohort

\begin{tabular}{lll}
\hline Demographic data & Number & Percent \\
\hline Clinical presentations* & & \\
Pelvic pain & $30 / 47$ & $63.82 \%$ \\
Pelvic and/or abdominal swelling & $6 / 47$ & $12.76 \%$ \\
Secondary infertility & $7 / 47$ & $14.89 \%$ \\
Postmenopausal bleeding & $4 / 47$ & $8.51 \%$ \\
Menstrual history & & \\
Premenopausal & $30 / 47$ & $63.82 \%$ \\
Postmenopausal & $17 / 47$ & $36.17 \%$ \\
\hline
\end{tabular}

*More than one clinical presentation in the same patient

The malignancy potential showed increased incidence in postmenopausal age with $P$ value $=0.039$.

There was no statistical significance between the lesions' size or T2 hydration signals and the pathological diagnosis. From the 47 cases, only 4 cases showed bilateral pathology $(8.5 \%)$ while the remaining 43 cases showed unilateral pathology $(91.5 \%)$. There was no statistical significance between the bilaterality of the lesion as well as the ancillary findings and final pathological diagnosis (Table 3).

Diffusion-weighted magnetic resonance imaging was done for all patients before contrast injection. Among our patients, $37 / 47$ cases (78.7\%) showed diffusion restriction and 10/47 cases (21.3\%) showed facilitated diffusion. The cutoff value of ADC value below which malignancy is expected is less than $1 \times 10^{-3} \mathrm{~mm}^{2} / \mathrm{s}$. There was statistical significance between ADC value and pathological diagnosis (Table 4).

As regards MRP, all semi-quantitative measurements were evaluated and correlated to the final histopathological results. There was a statistical significance between SI max, WIR and WOR and pathological diagnosis with higher sensitivity specificity and accuracy in relation to WOR. There was no statistical significant difference between MRE\% and pathological diagnosis (Tables 5 and 6).
Regarding the time signal intensity curves of MRP, we found that curves type 2 and 3 carry malignant probability with accuracy 95\%, sensitivity $88 \%$ and specificity 92\%. NPV 100\%

ROC curve analysis was used for prediction of malignancy depending on conventional-based diagnosis, DWI diagnosis, MRP and combined conventional MRI with DWI and with MRP.

There was a statistical significant difference between the three methods of diagnosis and the pathological diagnosis. However; the AUC, sensitivity, specificity and accuracy were higher on DWI and MRP-based diagnosis than those on the conventional-based diagnosis. Statistical correlation between results of conventional MRI, DWI and MRP to evaluate, the sensitivity, specificity, PPV, NPP and accuracy (Table 7).

Final pathological diagnosis after surgery was tabulated in Table 8.

\section{Discussion}

Adnexal masses are challenging diagnostic problems because of the overlapping imaging features between benign and malignant tumors. It is important to characterize an adnexal mass as accurately as possible to guide appropriate management and assure a good prognosis of surgical outcome. This is particularly important for young women who need fertility preservation $[12,13]$.

In our study, DWI-based diagnosis showed 100\% sensitivity while specificity remains low $88 \%$. Such low specificity elicited in our study can be explained by the presence of eight benign masses that mimicked malignancy. These masses included mature cystic teratomas, struma ovarii and benign sclerosing tumor. They all showed restricted diffusion and mean ADC values $0.8 \times 10^{-3} \mathrm{~mm}^{2} / \mathrm{s}, 1 \times 10^{-3} \mathrm{~mm}^{2} / \mathrm{s}$ and $0.9 \times 10^{-3} \mathrm{~mm}^{2} / \mathrm{s}$ respectively presenting (false positive cases) due to mixed cellularity of the lesions. The mean ADC values for benign lesions were $1.7 \pm 0.6 \times 10^{-3} \mathrm{~mm}^{2} / \mathrm{s}$ and for borderline and malignant lesions were $0.9 \pm$ $0.2 \times 10^{-3} \mathrm{~mm}^{2} / \mathrm{s}$ and $0.8 \pm 0.1$ respectively. The

Table 3 Conventional MRI findings with correlation to pathological final diagnosis

\begin{tabular}{|c|c|c|c|c|c|c|}
\hline & \multicolumn{3}{|c|}{ Pathological diagnosis } & \multirow[t]{3}{*}{$P$ value } & & \\
\hline & Benign (I) & Borderline (II) & Malignant (III) & & & \\
\hline & $N=18$ & $\mathrm{~N}=4$ & $\mathrm{~N}=25$ & & & \\
\hline Laterality: & & & & 0.118 & & \\
\hline Unilateral & 18(100\%) & $3(75 \%)$ & $22(88 \%)$ & | vs || & | vs ||| & \| vs III \\
\hline Bilateral & $0(0 \%)$ & $1(25 \%)$ & $3(12 \%)$ & 0.182 & 0.252 & 0.467 \\
\hline Ancillary findings & & & & 0.435 & & \\
\hline Yes & $5(27.8 \%)$ & $1(25 \%)$ & $12(48 \%)$ & | vs || & | vs ||| & II vs III \\
\hline No & $13(72.2 \%)$ & $3(75 \%)$ & $13(52 \%)$ & 1 & 0.219 & 0.606 \\
\hline
\end{tabular}

*Significant level at $P$ value $<0.05$ 
Table 4 Relation between ADC values of the lesion and final histopathological diagnosis

\begin{tabular}{|c|c|c|c|c|c|c|}
\hline & \multicolumn{3}{|c|}{ Pathological diagnosis } & \multirow[t]{3}{*}{$P$ value } & & \\
\hline & Benign (I) & Borderline (II) & Malignant (III) & & & \\
\hline & $N=18$ & $N=4$ & $N=25$ & & & \\
\hline ADC value & & & & $<0.001^{*}$ & & \\
\hline Range & $(0.9-3.2)$ & $(0.6-1.1)$ & $(0.5-1.1)$ & & & \\
\hline Mean & $1.7 \pm 0.6$ & $0.9 \pm 0.2$ & $0.8 \pm 0.1$ & | vs || & | vs ||| & II vs III \\
\hline Median & 1.7 & 0.9 & 0.8 & $0.008^{*}$ & $<0.001^{*}$ & 0.631 \\
\hline
\end{tabular}

*Significant level at $P$ value $<0.05$

cutoff value for ADC in malignancy is $\leq 1 \times 10^{-3}$ $\mathrm{mm}^{2} / \mathrm{s}$. This agreed with Thomassin-Naggara et al. [14] where they attributed the presence of low mean ADC values elicited by benign fibrous tumors as fibromas, Brenner tumors, and cystadenofibromas are due to dense network of collagen fibers within the extracellular matrix.

This is in contrary to the results of Zhang $\mathrm{P}$ et al. [15] who had high sensitivity and specificity of DWI. In his study that included 191 patients with 202 ovarian masses. They make exclusion of endometriomas, mature cystic teratomas and pure cystic adenomas from the analysis. Such exclusion may have elevated the specificity of the DWI. Yet, this was not applicable in our study because our analysis is a prospective evaluation and such action would subject our evaluation to a major bias.

MRP shows more effective results than simple dynamic contrast-enhanced MR sequence for qualitative assessment of enhancement [16, 17]. We performed MRP for 7 min as Gundogdu et al. [18]. Normal ovary demonstrated that the percentage of washout in the ovary was the highest at the 5th minute which is in concordance with Gundogdu et al. [18]. In contrary to Bernardin et al. [12] who performed a slow DCE sequence. They performed a 5-point dynamic run at $0,30,60,90$ and $120 \mathrm{~s}$.

In this study, we used the external myometrium as reference tissue as mentioned before by ThomassinNaggara et al. [19]. They performed quantitative DCE

Table 5 Correlation of measurements of MR perfusion with final histopathological diagnosis

\begin{tabular}{|c|c|c|c|c|c|c|}
\hline & \multicolumn{3}{|c|}{ Pathological diagnosis } & \multirow[t]{3}{*}{$P$ value } & & \\
\hline & Benign (I) & Borderline (II) & Malignant (III) & & & \\
\hline & $N=18$ & $N=4$ & $N=25$ & & & \\
\hline \multicolumn{4}{|l|}{$\overline{M R E}$} & 0.577 & & \\
\hline Range & $(50-190.8)$ & $(45-200.5)$ & $(39.7-141)$ & & & \\
\hline Mean \pm SD & $118.8 \pm 49.8$ & $118.2 \pm 80.6$ & $110.5 \pm 31.3$ & | vs || & | vs ||| & $\|$ vs II \\
\hline Median & 139 & 113.6 & 118.5 & 0.966 & 0.284 & 0.752 \\
\hline \multicolumn{4}{|l|}{ SI $\max$} & \multicolumn{3}{|l|}{$<0.001^{*}$} \\
\hline Range & $(66-1674.6)$ & $(1620.5-2630.5)$ & (1299.6-3729) & & & \\
\hline Mean \pm SD & $560.8 \pm 492.7$ & $1949.8 \pm 461.2$ & $1973.1 \pm 572.6$ & | vs || & | vs ||| & \| vs III \\
\hline Median & 513.4 & 1774.1 & 1900.2 & $0.003^{*}$ & $<0.001^{*}$ & 0.950 \\
\hline \multicolumn{4}{|l|}{ WIR } & \multicolumn{3}{|l|}{$<0.001^{*}$} \\
\hline Range & $(1.7-76.2)$ & $(11.6-113.2)$ & $(14-33.5)$ & & & \\
\hline Mean \pm SD & $15.3 \pm 15.7$ & $41.6 \pm 48$ & $23.2 \pm 5.4$ & | vs || & | vs ||| & \| vs II \\
\hline Median & 13 & 20.9 & 22 & 0.125 & $<0.001^{*}$ & 0.704 \\
\hline \multicolumn{7}{|l|}{ WOR } \\
\hline Range & $(0.6-6)$ & $(1-7.5)$ & $(6.5-17.2)$ & \multicolumn{3}{|l|}{$<0.001 *$} \\
\hline Mean \pm SD & $3.7 \pm 1.9$ & $3.1 \pm 3$ & $10.7 \pm 3.1$ & & & \\
\hline \multirow[t]{2}{*}{ Median } & 4.3 & 1.9 & 9.8 & | vs || & | vs ||| & $\|$ vs II \\
\hline & & & & 0.670 & $<0.001^{*}$ & $0.003^{*}$ \\
\hline
\end{tabular}

Kruska-Wallis test for nonparametric quantitative data between the three groups followed by Mann-Whitney test between each two groups. Fisher exact test for qualitative data between the groups

*Significant level at $P$ value $<0.05$ 
Table 6 ROC curve analysis for prediction of malignancy according to MR perfusion measurements in correlation with histopathological diagnosis

\begin{tabular}{llll}
\hline & SI max & WIR & WOR* $^{*}$ \\
\hline Sensitivity & 100 & 92 & 100 \\
Specificity & 94.44 & 94.44 & 100 \\
PPV & 96.2 & 95.8 & 100 \\
NPV & 100 & 89.5 & 100 \\
Accuracy & 97.67 & 93.02 & 100 \\
\hline
\end{tabular}

*Significant level at $P$ value $<0.05$

in 2010 to assess the physiological variations in myometrial vascularization throughout the menstrual cycle and to differentiate reproductive age from the postmenopausal state. The latter study found that there is no difference between the proliferative and the secretory phases, for all analyzed DCE parameters in the outer myometrium which makes it a suitable reference point. However, the outer myometrium for postmenopausal women displayed lower perfusion parameters than women of reproductive age and therefore this must be taken into account when enhancement curves are examined.

Other studies used pelvic striated muscles (psoas muscle) as reference tissue. However, striated muscles show variations in their perfusion in correlation with muscular atrophy. Some authors compared the diagnostic performance of both psoas muscle and myometrium in the DCE assessment of adnexal lesions. They found that both psoas muscles and myometrium are suitable internal references enabling high specificity for malignancy and benignity [20-22].

In our study, curve type I was found to be specific for benign ovarian tumors with $100 \%$ specificity. Curves type II and III were more in favor of borderline/malignant tumors. From all cases with curve type III, only one case was benign which was pathologically diagnosed as benign sclerosing tumor of the ovary. The explanation for such a result is that the sclerosing stromal tumors are highly vascular and under microscopy, it shows

Table 7 ROC curve analysis for prediction of malignancy among various MRI techniques in correlation to the final histopathological diagnosis

\begin{tabular}{lllll}
\hline & $\begin{array}{l}\text { Conventional } \\
\text { MRI }\end{array}$ & $\begin{array}{l}\text { Diffusion- } \\
\text { weighted } \\
\text { images }\end{array}$ & $\begin{array}{l}\text { MRI } \\
\text { perfusion }\end{array}$ & $\begin{array}{l}\text { Combined } \\
\text { conventional + DWI } \\
\text { or conventional + } \\
\text { MRI perfusion }\end{array}$ \\
\hline Sensitivity & $92 \%$ & $98 \%$ & $98 \%$ & $97 \%$ \\
Specificity & $61.11 \%$ & $83 \%$ & $83 \%$ & $92 \%$ \\
PPV & $76.7 \%$ & $90 \%$ & $90 \%$ & $90 \%$ \\
NPV & $84.6 \%$ & $98 \%$ & $98 \%$ & $98 \%$ \\
Accuracy & $79.1 \%$ & $93 \%$ & $93 \%$ & $93 \%$ \\
\hline
\end{tabular}

ectatic blood vessels which lead rapid rise in signal intensity than the myometrium with a well-defined shoulder. It should be mentioned here that the two excluded cases with tubo-ovarian abscess showed also curve type III. This also can be explained by the associated hyperemia and congestion of regional vessels.

The postulated theory of pathophysiology of tumor growth assumes that the tumors induce angiogenesis to be able to grow. Angionetic vessels formed by the tumors have large gaps between the endothelial cells, the endothelium and the basement membranes as well as between the basement membranes and the pericytes making the vessels hyperpermeable to many macromolecules. These properties can be exploited by DCE-MRI. MR contrast agents that leak slowly through the normal vasculature are able to pass more quickly through tumor vessels to produce differential enhancement. This results in a fast wash-in of contrast coupled with rapid washout and allows functional analysis of the tumor microcirculation [12].

In our study, the most perfusion parameters that showed significant difference between benign and malignant lesions were WOR, WIR and SImax $(P$ values < 0.001).The highest accuracy of the three measurements were WOR (100\%) followed by SImax (97\%) then WIR (93\%). The cutoff value for WOR in our study was $>6$ (lesions with WOR more than 6 is likely to be malignant). The PPV and NPV were $100 \%$ in Sohaib et al. study [20]. They found that malignant lesions show greater enhancement than benign lesions during the early phase of enhancement rather than the late phase of enhancement. This demonstrates the importance of SI max depicted in early dynamic phases in differentiating between benign and malignant lesions.

In our study, the cutoff value of SI max was $>1285$ (tumors with SImax more than that number is considered malignant). The sensitivity was $100 \%$ and specificity was $94 \%$. Those measurements do not agree with Dilks et al. [21] that suggested a threshold value of $>250$ for prediction of malignancy with a sensitivity and specificity $100 \%$. This difference can be explained by the unequal distribution of cases in our study. WIR showed less sensitivity, specificity, NPV, PPV and accuracy (92\%, 94\%, 89.5\%, 95.8\% and 93\% respectively) than WOR and SImax. Bernardin et al. [12] applied a cutoff value $>9.5$ for WIR where lesions with WIR mare than 9.5 are considered malignant. In our study, the cutoff value was higher than that value $(>17.9)$ and this also can be explained by the unequal distribution of cases with increased number of cases with hypervascular nature

Our study had several limitations. The unequal distribution of pathology included in the study. Some relatively common pathological entities were not included in 
Table 8 Final post-operative histopathological diagnosis

\begin{tabular}{llll}
\hline Type & Benign & Borderline & Malignant \\
\hline Epithelial tumors & & & Total \\
Serous & $3(6.3 \%)$ & $3(6.3 \%)$. & $6(12.7 \%)$. \\
Mucinous & $3(6.3 \%)$ & $1(2.1 \%)$ & $3(6.3 \%)$. \\
Brenner's & - & - & $2(4.2 \%)$. \\
Clear cell carcinoma & - & - & $1(2.1 \%)$. \\
Undifferentiated carcinoma & - & - & $1(2.1 \%)$. \\
Endometroid carcinoma & - & - & $1(2.1 \%)$. \\
Sex cord-stromal tumors & & - & - \\
Fibrothecoma/fibroma & $4(8.5 \%)$ & - & - \\
Benign sclerosing tumor & $1(2.1 \%)$ & - & $3(6.3 \%)$. \\
Granulosa cell tumor & - & - & - \\
Germ cell tumors & & - & - \\
Mature cystic teratoma & $4(8.5 \%)$ & - & $4(8.5 \%)$ \\
Struma ovarii & - & - & $4(8.5 \%)$. \\
Immature teratoma & - & & $17 \%)$ \\
Dysgerminoma & & & \\
\hline
\end{tabular}

the study as ovarian metastasis. Also, there is a small number of cases of ovarian epithelial borderline tumors included in the study. Most of the cases with epithelial borderline tumors are young age in the reproductive age, so that conservative rather than radical surgical approach may be recommended to preserve fertility. Further studies with a larger sample number and better pathological distribution are recommended. Also, the method of the ROI drawing that may be subject to human error, which may affect the performance. Some of our patients have complex masses that showed different tissue contents which may affect the selection of placement of ROI and influence readings.

\section{Conclusion}

MR diffusion and MR perfusion proved as accurate as each other in comparison with conventional MRI in diagnosis and characterization of malignant and benign ovarian tumors. They achieved high confidence levels. Improvements in the evaluation of SI max and WI and WO lead to the accurate evaluation of ovarian tumors. Standardized approaches of measurement and analysis approaches will lead to optimal diagnosis.

\section{Abbreviations}

ADC: Apparent diffusion coefficient; DWl: Diffusion-weighted imaging; MRD: magnetic resonance diffusion; MRP: Magnetic resonance perfusion; NPV: Negative predictive value; PPV: Positive predictive value; SD: Standard deviation

\section{Acknowledgements}

Not applicable.

\section{Authors' contributions}

All authors have read and approved the manuscript, and ensure that all of them read the manual and accept it. NE, MG, LM, OM and GB are the guarantor of integrity of the entire study, contributed to the study concept and design and data analysis. NE and GB contributed to the literature research and manuscript editing. $L M, O M$ and $G B$ contributed to the statistical analysis. NE, MG and GB contributed to the manuscript preparation.

\section{Funding}

Not applicable.

\section{Availability of data and materials}

All data are available for any confirmations or any explanations.

\section{Ethics approval and consent to participate}

This study was a prospective study approved by the ethical committee of our institution. The study population comprised 50 consecutive women who were suspected to have ovarian neoplastic mass. They underwent MRI imaging after meeting the inclusion criteria based on the clinical history and grayscale transabdominal and or transvaginal sonography findings. All patients included in this research gave written informed consent to publish the data contained within this study. Regarding patient less than 16 years old, written informed consent for the publication was given by their parent or legal guardian. There is no specific reference number for the approval of the ethical committee.

\section{Consent for publication}

All authors have read and approved the manuscript, and ensure that this is the case. All authors give their consent for publication after any corrections. We will do any reviewers comment before publications.

\section{Competing interests}

The authors declare that they have no competing interests.

Received: 4 October 2019 Accepted: 21 January 2020

Published online: 28 February 2020

\section{References}

1. Chornokur G, Amankwah EK, Schildkraut JM, Phelan CM (2013) Global ovarian cancer health disparities. Gynecol Oncol 129(1):258-264

2. Siegel R, Naishadham D, Jemal A (2013) Cancer statistics, 2013. CA Cancer J Clin 63(1):11-30 
3. Thomassin-Naggara I, Daraï E, Cuenod CA, Rouzier R, Callard P, Bazot M (2008) Dynamic contrast-enhanced magnetic resonance imaging: a useful tool for characterizing ovarian epithelial tumors. Magn Reson Imaging 28(1): $111-120$

4. Lutz AM, Willmann JK, Drescher CW, Ray P, Cochran FV, Urban N et al (2011) Early diagnosis of ovarian carcinoma: is a solution in sight? Radiology. 259(2):329-345

5. Cesario S (2010) Advances in the early detection of ovarian cancer: how to hear the whispers early. Nurs Womens Health 14(3):222-234

6. Medeiros $L R$, Freitas $L B$, Rosa DD, Silva FR, Silva $L S$, Birtencourt $L T$ et al (2011) Accuracy of magnetic resonance imaging in ovarian tumor: a systematic quantitative review. Am J Obstet Gynecol 204(1):67

7. Bazot M, Darai E, Nassar-Slaba J, Lafont C, Thomassin-Naggara I (2008) Value of magnetic resonance imaging for the diagnosis of ovarian tumors: a review. J Comput Assist Tomogr 32(5):712-723

8. Yankeelov TE, Gore JC (2009) Dynamic contrast enhanced magnetic resonance imaging in oncology: theory, data acquisition, analysis, and examples. Curr Med Imaging Rev 3(2):91-107

9. Tofts PS (2010) T1-weighted DCE imaging concepts: modelling, acquisition and analysis. Signal. 500(450):400

10. Kyriazi S, Kaye SB, De Souza NM (2010) Imaging ovarian cancer and peritoneal metastases_-current and emerging techniques. Nat Rev Clin Oncol 7(7):381-393

11. Li X, Hu J, Zhu LM, Sun XH, Sheng HQ, Zhai N, Hu XB, Sun CR, Zhao B (2015) The clinical value of dynamic contrast-enhanced MRI in differential diagnosis of malignant and benign ovarian lesions. Tumor Biol 36(7):55155522

12. Bernardin L, Dilks P, Liyanage S, Miquel M, Sahdev A, Rockall A (2012) Effectiveness of semi-quantitative multiphase dynamic contrast-enhanced MRI as a predictor of malignancy in complex adnexal masses: radiological and pathological correlation. Eur Radiol 22:880-890

13. Maarof RA, Abdelrahman AS, Habeeb DA (2018) Dynamic ContrastEnhanced Magnetic Resonance Imaging; A Useful Tool for Characterization and Assessment of Ovarian Masses. The Egyptian Journal of Hospital Medicine 71(2):2620-2625

14. Thomassin-Naggara I, Toussaint I, Perrot N, Rouzier R, Cuenod CA, Bazot M, Daraï E (2011) Characterization of complex adnexal masses: value of adding perfusion-and diffusion-weighted MR imaging to conventional MR imaging. Radiology 258(3):793-803

15. Zhang P, Li W, Chu C, Cui Y, Zhu M (2012) Diffusion-weighted MRl: a useful technique to discriminate benign versus malignant ovarian surface epithelial tumors with solid and cystic components. Abdominal Radiology 37(5):897-903

16. Forstner R, Thomassin-Naggara I, Cunha TM, Kinkel K, Masselli G, Kubik-Huch R, Spencer JA, Rockall A (2017) ESUR recommendations for MR imaging of the sonographically indeterminate adnexal mass: an update. Eur Radiol 27(6):2248-2257

17. Thomassin-Naggara I, Siles P, Balvay D, Cuenod CA, Carette MF, Bazot M (2013) MR perfusion for pelvic female imaging. Diagnostic and interventional imaging 94(12):1291-1298

18. Gundogdu S, Erdem CZ, Erdem LO, Bayar U (2006) Enhancement kinetics of normal ovaries on dynamic contrast-enhanced MR imaging. Eur J Obstet Gynecol Reprod Biol 129(1):60-64

19. Thomassin-Naggara I, Balvay D, Cuenod CA, Daraï E, Marsault C, Bazot M (2010) Dynamic contrast-enhanced MR imaging to assess physiologic variations of myometrial perfusion. Eur Radiol 20(4):984-994

20. Sohaib SA, Sahdev A, Trappen PV, Jacobs IJ, Reznek RH (2003) Characterization of adnexal mass lesions on MR imaging. Am J Roentgenol 180(5):1297-1304

21. Dilks P, Narayanan P, Reznek R, Sahdev A, Rockall A (2010) Can quantitative dynamic contrast-enhanced MRI independently characterize an ovarian mass? Eur Radiol 20(9):2176-2183

22. Tang YZ, Benardin L, BoothT C, Miquel ME, Dilks P, Sahdev A, Rockall AG (2014) Use of an internal reference in semi-quantitative dynamic contrastenhanced MRI (DCE MRI) of indeterminate adnexal masses. Br J Radiol 87(1043):20130730

\section{Publisher's Note}

Springer Nature remains neutral with regard to jurisdictional claims in published maps and institutional affiliations.

\section{Submit your manuscript to a SpringerOpen ${ }^{\circ}$ journal and benefit from:}

- Convenient online submission

- Rigorous peer review

- Open access: articles freely available online

- High visibility within the field

- Retaining the copyright to your article

Submit your next manuscript at $\boldsymbol{\nabla}$ springeropen.com 